\title{
FRACTAL DIMENSIONS OF SUBFRACTALS INDUCED BY SOFIC SUBSHIFTS
}

\author{
ELIZABETH SATTLER
}

\begin{abstract}
In this paper, we will consider subfractals of hyperbolic iterated function systems which satisfy the open set condition. The subfractals will consist of points associated with infinite strings from a subshift of finite type or sofic subshift on the symbolic space. We find that the zeros of the lower and upper topological pressure functions are lower and upper bounds, respectively, for the Hausdorff, packing, lower and upper box dimensions of the subfractal.
\end{abstract}

\section{INTRODUCTION}

One area of interest in fractal geometry is the study of properties which distinguish two distinct fractals. In particular, fractal dimensions, such as Hausdorff, box, and packing dimensions, have proven to be useful properties that in a sense, extend our usual notion of topological dimension. Numerous results exist for calculating the exact value of fractal dimensions of certain fractals of IFS type, such as self-similar IFSs [4,5,7], or finding bounds for the fractal dimensions for hyperbolic IFSs $[2,8]$. In this paper, we will focus on specific subsets of fractals of IFS type, namely, subfractals.

Clearly, not every subset of a fractal exhibits fractal-like properties; hence, one must provide a precise definition of a subfractal to produce a genuinely different fractal. For example, a subset of an IFS fractal may be a contracted copy of the entire fractal, which inherits most of the important properties (including fractal dimensions) from the whole fractal.

In this paper, we have chosen to identify a subfractal of an IFS type fractal by only considering those points associated with a subshift on the associated symbolic space. Unless stated otherwise, a subfractal will refer to a subshift-type subfractal for the remainder of this paper. If a subshift of finite type (SFT) or sofic subshift is chosen, we find that if the subshift does not have full Hausdorff dimension, then the Hausdorff dimension of the subfractal is strictly less than the Hausdorff dimension of the original fractal.

Let $\mathcal{A}$ denote a finite alphabet, $X$ denote the full shift, and $Y \subset X$ be a subshift. If $Y$ is a SFT, then there exists a matrix $A$ consisting only of 0 's and 1's which is associated with the subshift. The entries of $A$ are determined by finite strings in the subshift which are either allowed or not allowed to appear. We will use the notation $Y=X_{A}$ for the subshift associated with $A$.

Let $\mathcal{K} \subset \mathbb{R}^{n}$ be a compact subset and $\left(\mathcal{K} ; f_{1}, \ldots, f_{n}\right\}$ be an IFS with $f_{i}: \mathcal{K} \rightarrow \mathcal{K}$ for $1 \leq i \leq n$. The IFS $\left(\mathcal{K} ; f_{1}, \ldots, f_{n}\right\}$ is hyperbolic if for all $x, y \in \mathcal{K}$, there exists some constant $c$ such that $d\left(f_{i}(x), f_{i}(y)\right) \leq c d(x, y)$, for all $1 \leq i \leq n$. Let $F$ denote the attractor of this hyperbolic IFS (HIFS), i.e. $F$ is a non-empty, closed set with 
$f_{i}(F) \subset F$ for $1 \leq i \leq n$ and the smallest such set that satisfies these properties. By [5], we know that an attractor exists for any HIFS. Recall that an IFS satisfies the open set condition (OSC) if there exists a nonempty open subset $U \subset \mathcal{K}$ such that $f_{i}(U) \subset U$ and $f_{i}(U) \cap f_{J}(U)=\emptyset$ for $i \neq j$ and all $1 \leq i, j, \leq n$.

Now, let $\mathcal{F}_{X_{A}}$ be the collection of all points from the full fractal which are associated with a sequence in $X_{A}$, i.e. $x \in \mathcal{F}_{X_{A}}$ if there exists some $\omega=\omega_{1} \omega_{2} \ldots \in$ $X_{A}$ such that $x=\lim _{k \rightarrow \infty}\left[f_{\omega_{k}} \circ f_{\omega_{k-1}} \circ \cdots \circ f_{\omega_{1}}(y)\right], y \in \mathcal{K}$. Let $\rho(A)$ denote the spectral radius of a square matrix $A$. We prove the following:

Theorem (Main Theorem A). For a compact subset $\mathcal{K} \subset \mathbb{R}^{n}$, let $\left\{\mathcal{K} ; f_{i}: 1 \leq i \leq\right.$ $m\}$ be an HIFS which satisfies the OSC. Let $0<c_{i} \leq \bar{c}_{i}<1$ denote the constants such that $c_{i} d(x, y) \leq d\left(f_{i}(x), f_{i}(y)\right) \leq \bar{c}_{i} d(x, y)$ for all $1 \leq i \leq m$. Let $X_{A}$ be an SFT with an associated irreducible square $(0,1)$-matrix $A$. Let $\mathcal{F}_{X_{A}}$ denote the subfractal associated with the subshift. Then,

$$
h \leq \operatorname{dim}_{H}\left(\mathcal{F}_{X_{A}}\right) \leq H \text { and } h \leq \overline{\operatorname{dim}}_{B}\left(\mathcal{F}_{X_{A}}\right) \leq H,
$$

where $\rho\left(A S^{(h)}\right)=1=\rho\left(A \bar{S}^{(H)}\right), S$ and $\bar{S}$ are the corresponding diagonal matrices with appropriate constants $c_{i}$ and $\overline{c_{i}}$ on the diagonal and 0's elsewhere, $\left(S^{(h)}\right)_{i j}=$ $\left[S_{i j}\right]^{h}$ for all $1 \leq i, j \leq N$ and $\bar{S}^{(H)}$ is defined similarly.

Next, we turn our attention to a broader class of subshifts, the sofic subshifts. The class of sofic subshifts not only contains all SFTs but also all factors of SFTs. A common example of a sofic subshift which is not an SFT is the Golden Mean Shift, which has forbidden word list $F=\left\{101,10001, \ldots, 10^{2 k+1} 1, \ldots\right\}$ on the alphabet $\mathcal{A}=\{0,1\}$.

As in the case of subfractals induced by SFTs, a subfractal induced by a sofic subshift can be represented by a matrix $A_{\mathcal{G}}$; however, the entries of $A_{\mathcal{G}}$ consist of sums of contractive factors associated with finite, allowable strings from the subshift determined by an underlying labeled graph $\mathcal{G}$. Hence, we must alter the techniques we used for SFTs to compensate for the differences in the matrices associated with the subshifts. Let $\mathcal{L}$ denote the labeling with the lower contractive bounds and $\overline{\mathcal{L}}$ denote the labeling with the upper contractive bounds. See Section 5 for details on this labeling. We prove the following:

Theorem (Main Theorem B). For a compact subset $\mathcal{K} \subset \mathbb{R}^{n}$, let $\left\{\mathcal{K} ; f_{i}: 1 \leq i \leq\right.$ $m\}$ be an HIFS which satisfies the OSC. Let $0<c_{i} \leq \bar{c}_{i}<1$ denote the constants such that $c_{i} d(x, y) \leq d\left(f_{i}(x), f_{i}(y)\right) \leq \bar{c}_{i} d(x, y)$ for all $1 \leq i \leq m$. Let $X_{\mathcal{G}}$ be a sofic subshift wtih irreducible matrices $A=\left(a_{i j}\right)_{1 \leq i, j \leq k}$ and $\bar{A}=\left(\bar{a}_{i j}\right)_{1 \leq i, j \leq k}$ with $a_{i j}=\sum_{e_{i j}} \mathcal{L}\left(e_{i j}\right)$ and $\bar{a}_{i j}=\sum_{e_{i j}} \overline{\mathcal{L}}\left(e_{i j}\right)$. Then,

$$
h \leq \operatorname{dim}_{H}\left(\mathcal{F}_{X_{\mathcal{G}}}\right) \leq H \text { and } h \leq \overline{\operatorname{dim}}_{B}\left(\mathcal{F}_{X_{\mathcal{G}}}\right) \leq H,
$$

where $\rho\left(A_{h}\right)=1=\rho\left(A_{H}\right)$, and $A_{h}, A_{H}$ have entries $a_{i j}^{(h)}=\sum_{e_{i j}} \mathcal{L}\left(e_{i j}\right)^{h}, a_{i j}^{(H)}=$ $\sum_{e_{i j}} \overline{\mathcal{L}}\left(e_{i j}\right)^{H}$, respectively.

Remark 1. Theorem A will be split into Theorems 4.6 and 4.7 (for Hausdorff dimension bounds and upper box dimension bounds, respectively) in the case where $A$ is an irreducible matrix. Similarly, the case in which matrix $A_{\mathcal{G}}$ from Theorem $B$ is irreducible will be presented as Theorem 5.2. Theorem 6.3 will extend the results 
for Hausdorff dimension of subfractals defined by either a SFT or sofic subshift with a reducible matrix.

These results generalize previously proven results, including Theorems 1.1 and 1.2 below, which analyze different types of subfractals $[2,8]$. Following the notation and terminology in [8], we say that $A$ is primitive if there exists some integer $N$ such that $\left(A^{N}\right)_{i j}>0$ for all $1 \leq i, j \leq n$, where $\left(A^{N}\right)_{i j}$ denotes the $i j$-entry of $A^{N}$. A sequence of integers $\left(i_{l}\right)_{l \geq 1}$, where $i_{l} \in\{1, \ldots, n\}$, is said to be admissible if $(A)_{i_{l}, i_{l+1}} \neq 0$ for all $l \geq 1$. Let $F_{A}$ denote the collection of all points in $F$ which are associated with an admissible sequence with respect to $A$.

An HIFS is called disjoint if $f_{i}(F) \cap f_{j}(F)=\emptyset$ for all $i \neq j$ and $1 \leq i, j \leq n$. In [2], Ellis and Branton proved the following theorem.

Theorem 1.1. Let $F$ be the attractor of a disjoint HIFS $\left(\mathcal{K} ; f_{1}, \ldots, f_{n}\right)$, and let $A$ be a primitive $(0,1)$-matrix. Suppose that

$$
s_{i} d(x, y) \leq d\left(f_{i}(x), f_{i}(y)\right) \leq \overline{s_{i}} d(x, y),
$$

for all $x, y \in \mathcal{K}, 1 \leq i \leq n$, and for some constants $0<s_{i} \leq \overline{s_{i}}<1$. Then, $\operatorname{dim}_{H}\left(F_{A}\right) \leq u$, where $\rho\left(\overline{A \bar{S}^{u}}\right)=1$ and $\bar{S}$ is the diagonal matrix with diag $\left(\overline{s_{1}}, \ldots, \overline{s_{n}}\right)$.

In the same paper [2], Ellis and Branton made the following conjecture for the lower bound: $\operatorname{dim}_{H}\left(F_{A}\right) \geq l$ where $\rho\left(A S^{l}\right)=1$ and $S$ is a diagonal matrix with $s_{1}, \ldots, s_{n}$ on the diagonal and zeros elsewhere.

An $n \times n$ matrix $A$ is called irreducible if for all $1 \leq i, j \leq n$, there exists some finite sequence $\left(i_{l}\right)_{1 \leq l \leq m}$ with $i=i_{1}$ and $j=i_{m}$ such that $(\bar{A})_{i_{l}, i_{l+1}}(A)_{i_{l+1}, i_{l+2}}>0$ for $1 \leq l \leq m$. Every primitive matrix is irreducible, but there exist matrices which are irreducible and not primitive [6]. Let $N \geq 2$ and $\left\{\mathcal{K} ; f_{i j},(A)_{i j}: 1 \leq i \leq N\right\}$, where $f_{i j}: \mathcal{K} \rightarrow \mathcal{K}$ is a hyperbolic map for $1 \leq i, j \leq N$ and $A$ is an irreducible $(0,1)$-matrix. The system $\left\{\mathcal{K} ; f_{i j},(A)_{i j}: 1 \leq i \leq N\right\}$ is called a hyperbolic recurrent IFS.

A particular case of Roychowdhury's result below not only proves the conjecture proposed by Ellis and Branton, but also generalizes Theorem 1.1 by allowing the matrix $A$ to be irreducible and requiring the IFS to satisfy the OSC:

Theorem 1.2. Let $\left\{\mathcal{K} ; f_{i j},(A)_{i j}: 1 \leq i, j \leq N\right\}$ be a hyperbolic recurrent IFS which satisfies the open set condition and assume $A$ is irreducible. Let $F_{A}$ be the attractor of the system. Then,

$$
h \leq \operatorname{dim}_{H}\left(F_{A}\right) \leq H \text { and } h \leq \overline{\operatorname{dim}}_{H}\left(F_{A}\right) \leq H,
$$

where $h$ and $H$ are given by $\rho\left(\left((A)_{i j} s_{i j}^{h}\right)_{1 \leq i, j \leq N}\right)=1$ and $\rho\left(\left((A)_{i j} \bar{s}_{i j}\right)_{1 \leq i, j \leq N}\right)$ and $s_{i j}, \bar{s}_{i j}$ are given by $s_{i j} d(x, y) \leq d\left(f_{i j}(x), f_{i j}(y)\right) \leq \bar{s}_{i j} d(x, y)$.

Although it was not stated so, an attractor described above in Theorems 1.1 and 1.2 can be associated with an SFT defined by a list of forbidden words, each of length 2. Theorem A generalizes Theorem 1.1 completely in $\mathbb{R}^{n}$ and partially generelizes Theorem 1.2 by allowing the subfractal to be associated with any SFT, regardless of the length of the words in the forbidden word list. Furthermore, we extend the results to subfractals induced by a sofic subshift, which is a broader class than SFTs and, to our knowledge, is new. In the case of Hausdorff dimension, we 
remove the requirement that the associated matrices must be irreducible, and hence our results include even more subfractals induced by SFTs and sofic subshifts.

\section{BASIC DEFINITIONS AND BACKGROUND}

Let $\mathcal{K} \subset \mathbb{R}^{n}$ be a compact subset and $E \subseteq \mathcal{K}$. Letting $\overline{\mathcal{H}}_{\varepsilon}^{s}(E)=\inf _{\mathcal{U} \in \mathcal{O}} \sum_{U \in \mathcal{U}}(\operatorname{diam}(U))^{s}$, where $\mathcal{O}$ is the collection of all open $\varepsilon$-covers of $E$ and $s \geq 0$, the $s$-dimensional Hausdorff outer measure is defined to be $\overline{\mathcal{H}}^{s}=\lim _{\varepsilon \rightarrow 0} \overline{\mathcal{H}}_{\varepsilon}^{s}$. Restricting the outer measure to measurable sets, one defines the $s$-dimensional Hausdorff measure, $H^{s}$. The Hausdorff dimension of $E$, denoted $\operatorname{dim}_{\mathrm{H}}(E)$, is defined as the unique value of $s$ such that:

$$
H^{r}(E)= \begin{cases}0, & r>s \\ \infty, & r<s\end{cases}
$$

If $N_{r}(E)$ denotes the smallest number of sets of diameter $r$ that can cover $E$, the lower and upper box dimensions of $E$ are defined, respectively, as [3]:

$$
\underline{\operatorname{dim}}_{\mathrm{B}}(E)=\liminf _{r \rightarrow 0} \frac{\log N_{r}(E)}{-\log r} \text { and } \overline{\operatorname{dim}}_{\mathrm{B}}(E)=\limsup _{r \rightarrow 0} \frac{\log N_{r}(E)}{-\log r} .
$$

The following relationship between the fractal dimensions defined above are wellknown [3]:

$$
\operatorname{dim}_{H}(E) \leq \underline{\operatorname{dim}}_{B}(E) \leq \overline{\operatorname{dim}}_{B}(E) .
$$

Let $\mathcal{A}=\{1, \ldots, m\}$ be a finite alphabet. Let $\Omega_{n}$ denote the collection of all words on $\mathcal{A}$ of length $n$ and $\Omega_{*}=\bigcup_{k=1}^{\infty} \Omega_{k}$ denote the collection of all finite words of any finite length. Let $X$ denote the compact metric space of all infinite sequences on $\mathcal{A}$, equipped with the metric $d_{X}$ defined by $d_{X}(\omega, \tau)=\frac{1}{2^{k}}$ where $k=\min \left\{i: \omega_{i} \neq \tau_{i}\right\}$, for all $\omega=\omega_{1} \omega_{2} \ldots, \tau=\tau_{1} \tau_{2} \ldots \in X$. For $\omega \in \Omega_{*}$, let $\ell(\omega)$ denote the length of the word $\omega$. Let $\sigma: X \rightarrow X$ denote the shift map defined by $\sigma\left(\omega_{1} \omega_{2} \ldots\right)=\omega_{2} \omega_{3} \ldots$ for all $\omega=\omega_{1} \omega_{2} \ldots \in X$. We will also adopt the following notations:

$$
\begin{aligned}
\omega \tau & =\omega_{1} \ldots \omega_{n} \tau_{1} \ldots \tau_{m} \text { for } \omega \in \Omega_{n}, \tau \in \Omega_{m}, \\
\omega^{-} & =\omega_{1} \ldots \omega_{n-1} \text { for } \omega \in \Omega_{n}, \\
\left.\omega\right|_{n} & =\omega_{1} \ldots \omega_{n} \text { for all } \omega \in X .
\end{aligned}
$$

We will begin by focusing on specific subshifts, namely, subshifts of finite type (SFTs). An SFT, say Y, is defined by a finite list of forbidden words of finite length. A word $\tau \in \Omega_{n}$ is forbidden if it appears nowhere in $\omega$ for all $\omega \in Y$. Any word that is not forbidden is called an allowable word. Observe that for any list of forbidden words $F=\left\{x_{1}, \ldots, x_{k}\right\}, x_{i} \in \Omega_{*}$ for $1 \leq i \leq k$, there exists an integer $N$ such that $F$ can be rewritten as $F=\left\{y_{1}, \ldots, y_{l}\right\}$ where $y_{i} \in \Omega_{N}$ for $1 \leq i \leq l$. For more information on SFTs, refer to [6].

Let $\omega=\omega_{1} \ldots \omega_{k-1}, \xi=\xi_{1} \ldots \xi_{k-1} \in \Omega_{k-1}$. We say $\omega$ is compatible with $\xi$ if $\omega_{2} \ldots \omega_{k-1}=\xi_{1} \ldots \xi_{k-2}$. A compatible pair is a pair $(\omega, \xi) \in \Omega_{k-1} \times \Omega_{k-1}$, where $\omega$ is compatible with $\xi$. Let $\left(\Omega_{k-1} \times \Omega_{k-1}\right)_{\text {comp }}$ denote the collection of all compatible pairs $(\omega, \xi) \in \Omega_{k-1} \times \Omega_{k-1}$. Define an operation $*:\left(\Omega_{k-1} \times \Omega_{k-1}\right)_{\text {comp }} \rightarrow \Omega_{k}$ by $\omega * \xi=\omega_{1} \omega_{2} \ldots \omega_{k-1} \xi_{k-1}=\omega_{1} \xi_{1} \xi_{2} \ldots \xi_{k-1}$. 
Let $X_{F}$ be a SFT with forbidden words $F=\left\{\tau_{1}, \ldots, \tau_{l}\right\}$. Without loss of generality, we can assume $\tau_{i} \in \Omega_{k}$ for all $1 \leq i \leq l$. Let $W_{n}\left(X_{F}\right)$ denote all allowable words of length $n$ from $X_{F}$ for $n \geq 1$. If the subshift $X_{F}$ is clearly understood in context, we will typically write $W_{n}$. Let $W_{*}=\bigcup_{k=1}^{\infty} W_{k}$ denote the collection of all finite allowable strings.

Let $N=m^{k-1}$, where $m=|\mathcal{A}|$ and $\ell\left(\tau_{i}\right)=k$ for all $\tau_{i} \in F$. We will construct an $N$ x $N$ adjacency matrix $A$ as follows. Label the rows with all possible words (both allowable and forbidden) of length $k-1$, i.e. label the rows with $\left\{\omega_{1}, \ldots, \omega_{N}\right\}=$ $\Omega_{k-1}$. Label the correpsonding columns similarly. Let the entry be $a_{i j}=0$ if $\omega_{i}$ is not compatible with $\omega_{j}$ and $a_{i j}=0$ if $\omega_{i}$ is compatible with $\omega_{j}$ but $\omega_{i} * \omega_{j} \in F$. The entry $a_{i j}=1$ if $\omega_{i}$ is compatible with $\omega_{j}$ and $\omega_{1} * \omega_{j}$ is an allowable word.

For the sake of clarity, consider the following examples. First, consider the SFT on the alphabet $\mathcal{A}=\{1,2\}$ with forbidden word $F_{1}=\{22\}$. The associated matrix will be of the form:

$$
\left[\begin{array}{ll}
1 & 1 \\
1 & 0
\end{array}\right]
$$

Next, let us consider a SFT on the same alphabet $\mathcal{A}=\{1,2\}$ but with forbidden word list $F_{2}=\{112,211,222\}$. Since each forbidden word has length 3 , we will need to consider a $4 \times 4$ matrix since $\left|\Omega_{2}\right|=4$. We will choose the following labeling of rows: $R_{1} \rightarrow 11, R_{2} \rightarrow 12, R_{3} \rightarrow 21, R_{4} \rightarrow 22$. The corresponding matrix will be of the form:

$$
\left[\begin{array}{llll}
1 & 0 & 0 & 0 \\
0 & 0 & 1 & 1 \\
0 & 1 & 0 & 0 \\
0 & 0 & 1 & 0
\end{array}\right] .
$$

Here, the entries $a_{12}=a_{31}=a_{44}=0$ correspond to the forbidden words 112, 211, 222 , respectively. The entries $a_{13}=a_{14}=a_{21}=a_{22}=a_{33}=a_{34}=a_{41}=a_{42}=0$ correspond to pairs which are not compatible. The 1's in the matrix all correspond to compatible pairs which are also allowable words. We will use either $X_{A}$ or $X_{F}$ to denote the SFT.

To each such $N$ x $N$ adjacency matrix, we can associate a directed graph $G_{A}=$ $\left(V_{A}, E_{A}\right)$ where $V=\left\{v_{1}, v_{2}, \ldots, v_{N}\right\}$ and $E=\left\{e_{i, j}\right\}_{i, j=1}^{N}$ where $e_{i, j}$ is an edge from $v_{i}$ to $v_{j}$ if the entry $a_{i j}=1$ from $A$. A directed graph $G=(V, E)$ is called strongly connected if for any two vertices $v_{i}, v_{j} \in V$, there exists a path from $v_{i}$ to $v_{j}$.

Proposition 2.1. A matrix $A$ is irreducible iff it is associated with a graph $G_{A}$ which is strongly connected.

For details on Proposition 2.1, see [6]. By the Perron-Frobenius Theorem, we know that if $A$ is an irreducible matrix, then $A$ has a positive eigenvector $\mathbf{v}_{A}$ corresponding to a positive eigenvalue $\lambda_{A} \in \mathbb{R}$ such that $|\mu| \leq \lambda_{A}$ where $\mu$ is any eigenvalue of $A[6]$. For any non-negative $m \times m$ matrix $A$ with a positive eigenvector and corresponding positive eigenvalue $\lambda$, there exist constants $b_{0}, d_{0}>0$ 
such that

$$
b_{0} \lambda^{n} \leq \sum_{i, j=1}^{m}\left(A^{n}\right)_{i j} \leq d_{0} \lambda^{n}
$$

\section{Subfractals associated with a Subshift}

Let $\left\{\mathcal{K} ; f_{1}, \ldots f_{m}\right\}$ be the system defined in the statement of the main theorem, and let $\mathcal{F}$ denote the attractor of the HIFS. If $\mathcal{A}=\{1, \ldots, m\}$, where each letter $i$ corresponds to the map $f_{i}$ for $1 \leq i \leq m$, and $\omega=\omega_{1} \ldots \omega_{n} \in \Omega_{n}$, we will use the following notation:

$$
\begin{aligned}
& f_{\omega}=f_{\omega_{n}} \circ f_{\omega_{n-1}} \circ \cdots \circ f_{\omega_{1}} \\
& c_{\omega}=c_{\omega_{1}} c_{\omega_{2}} \cdots c_{\omega_{n}} .
\end{aligned}
$$

Define the associated coding map $\pi: X \rightarrow \mathcal{F}$ by $\pi(\omega)=\lim _{n \rightarrow \infty} f_{\left.\omega\right|_{n}}(\mathcal{K})$.

For each such IFS, we can define a subfractal of $\mathcal{F}$ by only considering the points associated with a word from a subshift. Let $X_{F}$ be a SFT and define $\mathcal{F}_{X_{F}}=\{\pi(\omega)$ : $\left.\omega \in X_{F}\right\}$.

As defined in section 2, fix an $N \times N$ adjacency matrix. Let $\Omega_{k-1}=\left\{\tau^{1}, \tau^{2}, \ldots, \tau^{N}\right\}$, $N=m^{k-1}$. Define two other $N \times N$ matrices, $S_{0}$ and $S$, as follows:

$$
S_{0}=\left[\begin{array}{cccc}
c_{\tau^{1}} & 0 & \cdots & 0 \\
0 & c_{\tau^{2}} & \cdots & 0 \\
\vdots & \vdots & \ddots & \vdots \\
0 & 0 & \cdots & c_{\tau^{N}}
\end{array}\right] \text { and } S=\left[\begin{array}{cccc}
c_{i_{1}} & 0 & \cdots & 0 \\
0 & c_{i_{2}} & \cdots & 0 \\
\vdots & \vdots & \ddots & \vdots \\
0 & 0 & \cdots & c_{i_{N}}
\end{array}\right]
$$

where $i_{j} \in \mathcal{A}$ for all $1 \leq j \leq N$ and the order of the $i_{j}^{\prime} s$ is chosen so that

$$
\sum_{i, j=1}^{N}\left(S_{0} A_{0} S\right)_{i, j}=\sum_{\omega \in \Omega_{k-1}} c_{\omega},
$$

with adjacency matrix $A_{0}$ associated with the full shift. Similarly, we define

$$
\bar{S}_{0}=\left[\begin{array}{cccc}
\bar{c}_{\tau^{1}} & 0 & \cdots & 0 \\
0 & \bar{c}_{\tau^{2}} & \cdots & 0 \\
\vdots & \vdots & \ddots & \vdots \\
0 & 0 & \cdots & \bar{c}_{\tau^{N}}
\end{array}\right] \text { and } \bar{S}=\left[\begin{array}{cccc}
\bar{c}_{i_{1}} & 0 & \cdots & 0 \\
0 & \bar{c}_{i_{2}} & \cdots & 0 \\
\vdots & \vdots & \ddots & \vdots \\
0 & 0 & \cdots & \bar{c}_{i_{N}}
\end{array}\right]
$$

For $t \in \mathbb{R}$, define

$$
S^{(t)}=\left[\begin{array}{cccc}
c_{i_{1}}^{t} & 0 & \cdots & 0 \\
0 & c_{i_{2}}^{t} & \cdots & 0 \\
\vdots & \vdots & \ddots & \vdots \\
0 & 0 & \cdots & c_{i_{N}}^{t}
\end{array}\right]
$$

and define $S_{0}^{(t)}, \bar{S}^{(t)}$, and $\bar{S}_{0}^{(t)}$ similarly.

Next, we will define a topological pressure function for calculating bounds for the fractal dimensions. Topological pressure functions have been used to find bounds for fractal dimensions of different types of fractal classes [8]. 
Definition 3.1. Let $X_{A}$ be a subshift. The lower topological pressure function of $\mathcal{F}_{X_{A}}$ is given by $P(t)=\lim _{n \rightarrow \infty} \frac{1}{n} \log \left(\sum_{\omega \in W_{n}} c_{\omega}^{t}\right)$. Similarly, we define the upper topological pressure function by $\bar{P}(t)=\lim _{n \rightarrow \infty} \frac{1}{n} \log \left(\sum_{\omega \in W_{n}} \bar{c}_{\omega}^{t}\right)$.

Proposition 3.2. The lower and upper topological pressure functions $P(t)$ and $\bar{P}(t)$ are strictly decreasing, convex, and continuous on $\mathbb{R}$.

Proof. We will show the proof for $P(t)$. The proof for $\bar{P}(t)$ follows similarly. Let $\delta>$ 0 . By using the fact that $c_{\omega} \leq c_{\max }^{n}$ for all $\omega \in W_{n}$, where $c_{\max }=\max _{1 \leq i \leq m}\left\{c_{i}\right\}$, we have:

$$
\begin{aligned}
P(t+\delta) & =\lim _{n \rightarrow \infty} \frac{1}{n} \log \left(\sum_{\omega \in W_{n}} c_{\omega}^{t+\delta}\right) \leq \lim _{n \rightarrow \infty} \frac{1}{n} \log \left(\sum_{\omega \in W_{n}} c_{\omega}^{t} c_{\text {max }}^{n \delta}\right) \\
& =\lim _{n \rightarrow \infty} \frac{1}{n} \log \left(c_{\max }^{n \delta} \sum_{\omega \in W_{n}} c_{\omega}^{t}\right)=\lim _{n \rightarrow \infty} \frac{1}{n}\left[n \delta \log \left(c_{\max }\right)\right]+P(t) \\
& =\delta \log \left(c_{\max }\right)+P(t)<P(t), \text { since } 0<c_{\max }<1 .
\end{aligned}
$$

Hence, $P(t)$ is strictly decreasing. If $t_{1}, t_{2} \in \mathbb{R}$ and $a_{1}, a_{2}>0$ with $a_{1}+a_{2}=1$, then, by Hölder's inequality, we have

$$
\begin{aligned}
P\left(a_{1} t_{1}+a_{2} t_{2}\right) & =\lim _{n \rightarrow \infty} \frac{1}{n} \log \left(\sum_{\omega \in W_{n}}\left(c_{\omega}\right)^{a_{1} t_{1}+a_{2} t_{2}}\right) \\
& =\lim _{n \rightarrow \infty} \frac{1}{n} \log \left[\sum_{\omega \in W_{n}}\left(\left(c_{\omega}\right)^{t_{1}}\right)^{a_{1}}\left(\left(c_{\omega}\right)^{t_{2}}\right)^{a_{2}}\right] \\
& \leq \lim _{n \rightarrow \infty} \frac{1}{n} \log \left[\sum_{\omega \in W_{n}}\left(c_{\omega}\right)^{t_{1}}\right]^{a_{1}}\left[\sum_{\omega \in W_{n}}\left(c_{\omega}\right)^{t_{2}}\right]^{a_{2}} \\
& =a_{1} P\left(t_{1}\right)+a_{2} P\left(t_{2}\right) .
\end{aligned}
$$

Hence, $P(t)$ is a convex function and strictly decreasing, and thus must be continuous.

Proposition 3.3. There is a unique value $h \in[0, \infty)$ such that $P(h)=0$.

Proof. If $t=0$,

$$
P(0)=\lim _{n \rightarrow \infty} \log \left(\sum_{\omega \in W_{n}} c_{\omega}^{0}\right)=\lim _{n \rightarrow \infty} \log \left(\left|W_{n}\right|\right) \geq 0 .
$$


Next, we will look at the case where $t \rightarrow \infty$.

$$
\begin{aligned}
P(t) & =\lim _{n \rightarrow \infty} \frac{1}{n} \log \left(\sum_{\omega \in W_{n}} c_{\omega}^{t}\right) \leq \lim _{n \rightarrow \infty} \frac{1}{n} \log \left(\sum_{\omega \in W_{n}} c_{\text {max }}^{n t}\right) \\
& =t \log \left(c_{\max }\right)+\lim _{n \rightarrow \infty} \frac{1}{n} \log \left(\left|W_{n}\right|\right) \leq t \log \left(c_{\max }\right)+\lim _{n \rightarrow \infty} \frac{1}{n} \log \left(m^{n}\right) \\
& =t \log \left(c_{\max }\right)+\log (m) .
\end{aligned}
$$

Since $0<c_{\max }<1$, we must have $\left[t \log \left(c_{\max }\right)+\log (m)\right] \rightarrow-\infty$ as $t \rightarrow \infty$, and hence $\lim _{t \rightarrow \infty} P(t)=-\infty$. By Proposition 3.2, there exists a unique value $h$ such that $P(h)=0$.

Following the same steps as in the proof above, we have:

Proposition 3.4. There is a unique value $H \in[0, \infty)$ such that $\bar{P}(H)=0$.

Proposition 3.5. Let $h$ and $H$ be the unique values such that $P(h)=0=\bar{P}(H)$. Then, $h \leq H$.

Proof. Assume that $h>H$. Then, $\bar{P}(h)<\bar{P}(H)=0$. We also know that $c_{\omega} \leq \bar{c}_{\omega}$ for all $\omega \in W_{n}$. Hence,

$$
0=P(h)=\lim _{n \rightarrow \infty} \frac{1}{n} \log \left(\sum_{\omega \in W_{n}} c_{\omega}^{h}\right) \leq \lim _{n \rightarrow \infty} \frac{1}{n} \log \left(\sum_{\omega \in W_{n}} \bar{c}_{\omega}^{h}\right)=\bar{P}(h)<0,
$$

which is a contradiction. Hence, $h \leq H$.

Lemma 3.6. Let $X_{A}$ be an $S F T$ associated with matrix $A$, and let $\left\{\mathcal{K} ; f_{i}: 1 \leq\right.$ $i \leq m\}$ be an HIFS. Let $S_{0}$ and $S$ be matrices associated with the subfractal $\mathcal{F}_{X_{A}}$, as above. Then, the associated lower and upper topological pressure functions $P(t)$ and $\bar{P}(t)$ can be written, respectively, as

$$
\begin{gathered}
P(t)=\lim _{n \rightarrow \infty} \frac{1}{n} \log \left(\sum_{i, j=1}^{N}\left[S_{0}^{(t)}\left(A S^{(t)}\right)^{n-k+1}\right]_{i, j}\right) \text { and } \\
\bar{P}(t)=\lim _{n \rightarrow \infty} \frac{1}{n} \log \left(\sum_{i, j=1}^{N}\left[\bar{S}_{0}^{(t)}\left(A \bar{S}^{(t)}\right)^{n-k+1}\right]_{i, j}\right) .
\end{gathered}
$$

Proof. Recall that if $F$ is a list of forbidden words, all of length $k$, then $A$ is an $N$ x $N$ matrix, where $N=\left|\Omega_{k-1}\right|=m^{k-1}$. We will prove the assertion by induction. First, the nonzero entries of $A$ correspond to the allowable words of length $k$. Hence, by definition of $A, S_{0}$, and $S$, we have

$$
\sum_{i, j=1}^{N}\left[S_{0} A S\right]_{i j}=\sum_{\omega \in W_{k}} c_{\omega}
$$

Now, assume that $\sum_{i, j=1}^{N}\left[S_{0}(A S)^{n}\right]_{i j}=\sum_{\omega \in W_{n+k-1}} c_{\omega}$ for some $n>1$. The entries of $S_{0}(A S)^{n}$ consist of sums of contractive factors associated with allowable words of length $n+k-1$. Now, consider the matrix $S_{0}(A S)^{n}(A S)$. By the definition of $A$ and $S$, this multiplication will result in entries consisting of sums of contractive 
factors associated with allowable words of length $n+k$. Since $S_{0}(A S)^{n}$ contains all allowable words of length $n+k-1$, then we must have $\sum_{i, j=1}^{N}\left[S_{0}(A S)^{n+1}\right]_{i j}=$ $\sum_{\omega \in W_{n+k}} c_{\omega}$. Hence,

$$
P(t)=\lim _{n \rightarrow \infty} \frac{1}{n} \log \left(\sum_{\omega \in W_{n}} c_{\omega}^{t}\right)=\lim _{n \rightarrow \infty} \frac{1}{n} \log \left(\sum_{i, j=1}^{N}\left[S_{0}^{(t)}\left(A S^{(t)}\right)^{n-(k-1)}\right]_{i j}\right) .
$$

The proof follows similarly for the upper topological pressure function.

\section{MAIN THEOREM FOR SFTS}

We begin with a technical lemma that will provide bounds needed for the main result.

Lemma 4.1. Let $S_{0}, A$, and $S$ be defined as in Section 3, where $A$ is an irreducible matrix. Then, for any $t>0$, there exist positive constants $K, L$ such that

$$
c_{\min }^{(k-1) t} K \lambda_{A S^{(t)}}^{n} \leq \sum_{i, j=1}^{N}\left[S_{0}^{(t)}\left(A S^{(t)}\right)^{n}\right]_{i, j} \leq c_{\max }^{(k-1) t} L \lambda_{A S^{(t)}}^{n},
$$

where $c_{\min }=\min _{1 \leq i \leq m}\left\{c_{i}\right\}, c_{\max }=\max _{1 \leq i \leq m}\left\{c_{i}\right\}, \lambda_{A S^{(t)}}$ is the maximal eigenvalue of $A S^{(t)}$.

Proof. Notice that for every non-zero entry of $S_{0}$, we have $c_{\min }^{k-1} \leq\left(S_{0}\right)_{i j} \leq c_{\max }^{k-1}$, $1 \leq i, j \leq N$. Hence, by the Perron-Frobenius Theorem, we have constants $K$ and $L$ such that

$$
\begin{gathered}
c_{\min }^{(k-1) t} K \lambda_{A S^{t}}^{n} \leq c_{\min }^{(k-1) t} \sum_{i, j=1}^{N}\left[\left(A S^{(t)}\right)^{n}\right]_{i, j} \leq \sum_{i, j=1}^{N}\left[S_{0}^{(t)}\left(A S^{(t)}\right)^{n}\right]_{i, j} \\
\leq c_{\max }^{(k-1) t} \sum_{i, j=1}^{N}\left[\left(A S^{(t)}\right)^{n}\right]_{i, j} \leq c_{\max }^{(k-1) t} L \lambda_{A S^{(t)}}^{n}
\end{gathered}
$$

Remark 2. By Lemma 4.1, one can show that, for fixed value $t \in[0, \infty]$,

$$
\begin{aligned}
P(t) & =\lim _{n \rightarrow \infty} \frac{1}{n} \log \left(\sum_{i, j=1}^{N}\left[S_{0}^{(t)}\left(A S^{(t)}\right)^{n}\right]_{i j}\right) \\
& \leq \lim _{n \rightarrow \infty} \frac{1}{n} \log \left(c_{\text {max }}^{(k-1) t} L \lambda_{A S^{(t)}}^{n}\right)=\log \left(\lambda_{A S^{(t)}}\right)=\log \left(\rho\left(A S^{(t)}\right),\right.
\end{aligned}
$$

where $\rho\left(A S^{(t)}\right)$ denotes the spectral radius of $A S^{(t)}$. Similarly, we can show that $\log \left(\rho\left(A S^{(t)}\right)\right) \leq P(t)$, and hence $P(t)=\log \left(\rho\left(A S^{(t)}\right)\right.$. Therefore, the unique value $h$ such that $P(h)=0$ is also the value of $h$ such that $\rho\left(A S^{(h)}\right)=1$. Analogously, we can show that the value $H$ such that $\bar{P}(H)=0$ is also the value of $H$ that satisfies $\rho\left(A \bar{S}^{(H)}\right)=1$. 
Proposition 4.2. Let $h$ be the unique zero of the lower topological pressure function. There exist positive constants $K_{0}, L_{0}$ such that

$$
K_{0} \leq \sum_{\omega \in W_{n}} c_{\omega}^{h} \leq L_{0}
$$

for all $n \geq 1$.

Proof. Let $s<h$. Then, $P(s)>P(h)=0$. So, we have

$$
\begin{aligned}
0<P(s) & =\lim _{p \rightarrow \infty} \frac{1}{n p} \log \left(\sum_{\omega \in W_{n p}} c_{\omega}^{s}\right) \leq \lim _{p \rightarrow \infty} \frac{1}{n p} \log \left(\sum_{\omega \in W_{n}} c_{\omega}^{s}\right)^{p} \\
& =\frac{1}{n} \log \left(\sum_{\omega \in W_{n}} c_{\omega}^{s}\right) .
\end{aligned}
$$

Hence, $\sum_{\omega \in W_{n}} c_{\omega}^{s}>1$, and it follows that $\sum_{\omega \in W_{n}} c_{\omega}^{h} \geq 1$.

Now, assume that $s>h$. Then, $0=P(h)>P(s)$. So, by Lemma 4.1, we have

$$
\begin{aligned}
0>P(s) & =\lim _{p \rightarrow \infty} \frac{1}{n p} \log \left(\sum_{\omega \in W_{n p}} c_{\omega}^{s}\right)=\lim _{p \rightarrow \infty} \frac{1}{n p} \log \left(\sum_{i, j=1}^{N}\left[S_{0}^{(s)}\left(A S^{(s)}\right)^{n p}\right]_{i, j}\right) \\
& \geq \lim _{p \rightarrow \infty} \frac{1}{n p} \log \left(c_{\min }^{(k-1) s} K \lambda_{A S^{(s)}}^{n p}\right)=\frac{1}{n} \log \left(\lambda_{A S^{(s)}}^{n}\right) \\
& \geq \frac{1}{n} \log \left(\frac{1}{L c_{\text {max }}^{(k-1) s}} \sum_{i, j=1}^{N}\left[S_{0}^{(s)}\left(A S^{(s)}\right)^{n}\right]_{i, j}\right)=\frac{1}{n} \log \left(\frac{1}{L c_{\text {max }}^{(k-1) s}} \sum_{\omega \in W_{n}} c_{\omega}^{s}\right) .
\end{aligned}
$$

Hence, $\sum_{\omega \in W_{n}} c_{\omega}^{s}<L c_{\max }^{(k-1) s}$, which implies that $\sum_{\omega \in W_{n}} c_{\omega}^{h} \leq L c_{\max }^{(k-1) h}$.

Following similar steps in the proof of Proposition 4.2, we have:

Proposition 4.3. Let $H$ be the unique zero of the upper topological pressure function. There exist positive constants $K_{1}, L_{1}$ such that

$$
K_{1} \leq \sum_{\omega \in W_{n}} \bar{c}_{\omega}^{H} \leq L_{1} .
$$

In order to show that $h$ is a lower bound for $\operatorname{dim}_{H}(\mathcal{F})$, we will utilize the uniform mass distribution principle from Falconer [4]. Hence, we must define an appropriate Borel probability measure to satisfy the principle. Let $h$ be the unique value such that $P(h)=0$. Let $\omega \in \Omega_{n}$ and let $\llbracket \omega \rrbracket=\left\{\tau \in \Omega_{\infty}: \tau_{i}=\omega_{i}, 1 \leq i \leq n\right\}$ be the cylinder set corresponding to $\omega$. We will use the fact that $c_{\omega \tau}=c_{\omega} c_{\tau}$. Define

$$
\nu_{n}(\llbracket \omega \rrbracket)=\frac{\sum_{\omega \tau \in W_{n+\ell(\omega)}} c_{\omega \tau}^{h}}{\sum_{\tau \in W_{n+\ell(\omega)}} c_{\tau}^{h}} .
$$

For all $n \geq 1$ and any $\omega \in W_{*}$, we have by Proposition 4.2,

$$
0 \leq \frac{\sum_{\omega \tau \in W_{n+\ell(\omega)}} c_{\omega \tau}^{h}}{L_{0}} \leq \nu_{n}(\llbracket \omega \rrbracket) \leq \frac{c_{\omega}^{h} \sum_{\tau \in W_{n}} c_{\tau}^{h}}{\sum_{\tau \in W_{n}+\ell(\omega)} c_{\tau}^{h}} \leq \frac{L_{0}}{K_{0}} c_{\omega}^{h}<\infty .
$$


Hence, for all $\omega \in W_{*}, \operatorname{Lim}_{n \rightarrow \infty} \nu_{n}(\llbracket \omega \rrbracket)$ exists, where Lim denotes the Banach limit. Let $\nu(\llbracket \omega \rrbracket)=\operatorname{Lim}_{n \rightarrow \infty} \nu_{n}(\llbracket \omega \rrbracket)$.

Also, notice that

$$
\begin{aligned}
\sum_{i=1}^{m} \nu(\llbracket \omega i \rrbracket) & =\operatorname{Lim}_{n \rightarrow \infty} \sum_{i=1}^{m} \frac{\sum_{\omega i \tau \in W_{n+\ell(\omega i)}} c_{\omega i \tau}^{h}}{\sum_{\tau \in W_{n+\ell(\omega i)}} c_{\tau}^{h}} \\
& =\operatorname{Lim}_{n \rightarrow \infty} \frac{\sum_{\omega \tau \in W_{n+1+\ell(\omega)}} c_{\omega \tau}^{h}}{\sum_{\tau \in W_{n+1+\ell(\omega)}} c_{\tau}^{h}}=\nu(\llbracket \omega \rrbracket) .
\end{aligned}
$$

Hence, by applying Kolmogorov extension theorem, we can extend $\nu$ to a unique Borel probability measure $\gamma$ on $X_{A}$. Let $\mu_{h}=\gamma \circ \pi^{-1}$, where $\pi$ is the coding map. Hence, $\mu_{h}$ is supported on $\mathcal{F}_{X_{A}}$.

Corollary 4.4. There exist constants $K_{0}, L_{0}>0$ such that

$$
\mu_{h}\left(f_{\omega}(\mathcal{K})\right) \leq \frac{L_{0}}{K_{0}} c_{\omega}^{h} .
$$

Proof. By definition of $\mu_{h}$ and Proposition 4.2, we have

$$
\mu_{h}\left(f_{\omega}(\mathcal{K})\right)=\nu(\llbracket \omega \rrbracket)=\frac{\sum_{\omega \tau \in W_{n}} c_{\omega \tau}^{h}}{\sum_{\tau \in W_{n+\ell(\omega)}} c_{\tau}^{h}} \leq \frac{c_{\omega}^{h} \sum_{\tau \in W_{n}} c_{\tau}^{h}}{\sum_{\tau \in W_{n+|\omega|}} c_{\tau}^{h}} \leq c_{\omega}^{h} \frac{L_{0}}{K_{0}} .
$$

Proposition 4.5. For $0<r<1$ and $x \in \mathcal{F}$, the ball $B(x, r)$ intersects at most $M$ elements of $\mathcal{U}_{r}=\left\{f_{\omega}(\mathcal{K}):\left|f_{\omega}(\mathcal{K})\right| \leq r<\left|f_{\omega^{-}}(\mathcal{K})\right|\right\}$, where $M$ is finite and independent of $r$.

Proof. Let $0<r<1$ and $x \in \mathcal{F}$. Let $W_{r}=\left\{\omega \in W_{*}: f_{\omega}(\mathcal{K}) \cap B(x, r) \neq \emptyset, f_{\omega}(\mathcal{K}) \in\right.$ $\left.\mathcal{U}_{r}\right\}$ and $\left|W_{r}\right|=M$. Let $y \in B(x, r)$ and $z \in f_{\omega}(\mathcal{K})$ where $\omega \in W_{r}$. Notice that

$$
d(y, z) \leq|B(x, r)|+\left|f_{\omega}(\mathcal{K})\right| \leq 3 r .
$$

Hence, $\left\{f_{\omega}(\mathcal{K}): \omega \in W_{r}\right\} \subset B(x, 3 r)$. For any $f_{\omega}(\mathcal{K}) \in \mathcal{U}_{r}$, we have

$$
\left|f_{\omega}(\mathcal{K})\right| \geq c_{\min }\left|f_{\omega^{-}}(\mathcal{K})\right|>c_{\text {min }} r .
$$

Due to the open set condition, there exists a ball $B_{a}$ of radius $a>0$ such that $B_{a} \subset \mathcal{K}$ and $f_{\omega}\left(B_{a}\right) \cap f_{\tau}\left(B_{a}\right)=\emptyset$ for $\omega, \tau \in W_{r}$. For each $\omega \in W_{r}$, we have $f_{\omega}\left(B_{a}\right) \subset f_{\omega}(\mathcal{K})$. Let $m$ denote Lebesgue measure on $\mathcal{K}$. Since the balls are disjoint and contained in $B(x, 3 r)$, we have

$$
\sum_{\omega \in W_{r}} m\left(f_{\omega}\left(B_{a}\right)\right) \leq m(B(x, 3 r)) .
$$

Using the fact that $\left|f_{\omega}(\mathcal{K})\right|>c_{\text {min }} r$, we have

$$
M \cdot m\left(B\left(x, a c_{\min } r\right)\right) \leq m(B(x, 3 r) .
$$

Hence, $M \leq \frac{m(B(x, 3 r)}{m\left(B\left(x, a c_{\min } r\right)\right)}$. Since the ratio compares concentric balls, each with a radius equal to a constant multiple of $r$, we can let $M \leq\left\lceil\frac{m(B(x, 3 r))}{m\left(B\left(x, c_{m i n} r\right)\right)}\right\rceil<\infty$, which satisfies the assertion of the proposition. 
Theorem 4.6. Let $h, H$ be the unique values such that $P(h)=0=\bar{P}(H)$. Then, $h \leq \operatorname{dim}_{H}(\mathcal{F}) \leq H$.

Proof. Let $\mathcal{U}_{n}=\left\{f_{\omega}(\mathcal{K}): \omega \in W_{n}\right\}$. Notice that $\mathcal{U}_{n}$ is a cover for all $n \geq 1$. Hence, by Proposition 4.3 , we have

$$
\begin{aligned}
\mathcal{H}^{H}(\mathcal{F}) & =\lim _{\varepsilon \rightarrow 0} \inf _{\mathcal{E}} \sum_{E \in \mathcal{E}}|E|^{H} \leq \lim _{n \rightarrow \infty} \sum_{\omega \in W_{n}}\left|f_{\omega}(\mathcal{K})\right|^{H} \\
& \leq \lim _{n \rightarrow \infty} \sum_{\omega \in W_{n}}|\mathcal{K}|^{H} \bar{c}_{\omega}^{H} \leq|\mathcal{K}|^{H} \cdot L_{1}<\infty .
\end{aligned}
$$

Thus, $\operatorname{dim}_{h}(\mathcal{F}) \leq H$. Let $r>0$ and $B(x, r)$ be a ball centered at $x \in \mathcal{F}$. By Proposition 4.5, $B(x, r)$ intersects at most $M$ elements of the cover $\mathcal{U}_{r}$. Let $\mathcal{U}_{M}$ denote the subset of $\mathcal{U}_{r}$ consisting of all elements that intersect $B(x, r)$ and $W_{M}$ denote all allowable words associated with an element of $\mathcal{U}_{M}$. By Corollary 4.4, we have

$$
\begin{aligned}
\frac{\mu_{h}(B(x, r))}{r^{h}} & \leq \frac{\sum_{f_{\omega}(\mathcal{K}) \in \mathcal{U}_{M}} \mu_{h}\left(f_{\omega}(\mathcal{K})\right)}{r^{h}} \leq \frac{\sum_{\omega \in W_{M}} \frac{L_{0}}{K_{0}} c_{\omega}^{h}}{r^{h}} \\
& \leq \frac{M \frac{L_{0}}{K_{0}}|\mathcal{K}|^{-h} r^{h}}{r^{h}}=M \frac{L_{0}}{K_{0}}|\mathcal{K}|^{-h} .
\end{aligned}
$$

Hence, $\limsup _{r \rightarrow 0} \frac{\mu_{h}(B(x, r))}{r^{h}} \leq M \frac{L_{0}}{K_{0}}|\mathcal{K}|^{-h}<\infty$. By the uniform mass distribution principle [4], we have $\mathcal{H}^{h}(\mathcal{F}) \geq \frac{M \frac{L_{0}}{K_{0}}|\mathcal{K}|^{-h}}{\mu_{h}(\mathcal{F})}>0$. Thus, $\operatorname{dim}_{H}(\mathcal{F}) \geq h$.

Theorem 4.7. Let $h, H$ be the unique values such that $P(h)=0=\bar{P}(H)$. Then, $h \leq \overline{\operatorname{dim}}_{B}(\mathcal{F}) \leq H$.

Proof. The following relationship between Hausdorff and box dimensions is wellknown:

$$
\operatorname{dim}_{H}(\mathcal{F}) \leq \underline{\operatorname{dim}}_{B}(\mathcal{F}) \leq \overline{\operatorname{dim}}_{B}(\mathcal{F}) .
$$

Hence, it suffices to show that $\overline{\operatorname{dim}}_{B}(\mathcal{F}) \leq H$. Let $\mathcal{U}_{r}=\left\{f_{\omega}(\mathcal{K}):\left|f_{\omega}(\mathcal{K})\right| \leq r<\right.$ $\left.\left|f_{\omega^{-}}(\mathcal{K})\right|\right\}, k=\min \left\{|\omega|: f_{\omega}(\mathcal{K}) \in \mathcal{U}_{r}\right\}$, and $\mathcal{O}_{k}=\left\{f_{\omega}(\mathcal{K}): \omega \in W_{k}\right\}$. Notice that $\bigcup_{\mathcal{N}} f_{\omega}(\mathcal{K}) \subseteq \bigcup_{f_{\omega}} f_{\omega}(\mathcal{K})$. Hence, by Proposition 4.4, we have $f_{\omega}(\mathcal{K}) \in \mathcal{U}_{r}$

$$
\sum_{f_{\omega}(\mathcal{K}) \in \mathcal{U}_{r}}\left|f_{\omega}(\mathcal{K})\right|^{H} \leq \sum_{f_{\omega}(\mathcal{K}) \in \mathcal{O}_{k}}\left|f_{\omega}(\mathcal{K})\right|^{H} \leq|\mathcal{K}|^{H} \sum_{\omega \in W_{k}} \bar{c}_{\omega}^{H} \leq|\mathcal{K}|^{H} L_{1} .
$$

Also, for $f_{\omega}(\mathcal{K}) \in \mathcal{U}_{r}$,

$$
\left|f_{\omega}(\mathcal{K})\right| \geq\left|f_{\omega^{-}}(\mathcal{K})\right| \cdot c_{\text {min }}>r c_{\text {min }} .
$$

Let $N_{r}(\mathcal{F})$ denote the smallest number of sets of diameter at most $r$ which form a cover of $\mathcal{F}$. Then,

$$
\left(r c_{\text {min }}\right)^{H} N_{r}(\mathcal{F}) \leq\left|f_{\omega}(\mathcal{K})\right|^{H} N_{r}(\mathcal{F}) \leq \sum_{f_{\omega}(\mathcal{K}) \in \mathcal{U}_{r}}\left|f_{\omega}(\mathcal{K})\right|^{H} \leq|\mathcal{K}|^{H} L_{1} .
$$

Hence, $N_{r}(\mathcal{F}) \leq\left(r c_{\text {min }}\right)^{-H}|\mathcal{K}|^{H} L_{1}$, and thus

$$
\frac{\log \left(N_{r}(\mathcal{F})\right)}{-\log (r)} \leq \frac{\log \left(L_{1}|\mathcal{K}|^{H}\right)-H \log \left(r c_{\text {min }}\right)}{-\log (r)}=\frac{\log \left(L_{1}|\mathcal{K}|^{H}\right)}{-\log (r)}+\frac{H \log \left(c_{\text {min }}\right)}{\log (r)}+H
$$


By the definition of upper box dimension, we have

$\overline{\operatorname{dim}}_{B}(\mathcal{F})=\limsup _{r \rightarrow 0} \frac{\log \left(N_{r}(\mathcal{F})\right)}{-\log (r)} \leq \limsup _{r \rightarrow 0}\left[\frac{\log \left(L_{1}|\mathcal{K}|^{H}\right)}{-\log (r)}+\frac{H \log \left(c_{m i n}\right)}{\log (r)}\right]+H=H$.

Remark 3. For $E \subset \mathcal{K}$, the following inequalities are well-known:

$$
\operatorname{dim}_{H}(E) \leq \operatorname{dim}_{P}(E) \leq \overline{\operatorname{dim}}_{B}(E) \text { and } \operatorname{dim}_{H}(E) \leq \underline{\operatorname{dim}}_{B} \leq \overline{\operatorname{dim}}_{B}(E)
$$

where $\operatorname{dim}_{P}(E)$ denote the packing dimension of $E$. For more information on packing dimension, see [1]. Hence, we have also shown that

$$
h \leq \operatorname{dim}_{P}(\mathcal{F}) \leq H \text { and } h \leq \underline{\operatorname{dim}}_{B}(\mathcal{F}) \leq H .
$$

\section{Main theorem for Sofic Subshifts}

In this section, we will extend the assertions from Theorems 4.6 and 4.7 to sofic subshifts. Recall that SFTs are sofic subshifts, but there exist sofic subshifts which cannot be represented as a SFT. A common charaterization of a sofic shift $(Y, \sigma)$ is that it must be a factor of some $\mathrm{SFT}$, some $(X, \sigma)$. That is, there exists a continuous map $\psi: X \rightarrow Y$ such that $\sigma \circ \psi=\psi \circ \sigma$.

We adopt the following definitions from [6]. Let $\mathcal{G}=(G, \mathcal{L})$ be a labeled graph, consisting of a graph $G$ with edge set $\mathcal{E}$ and a labeling $\mathcal{L}: \mathcal{E} \rightarrow \mathcal{A}$, where $\mathcal{A}$ is the finite alphabet. A subset $X$ of the full shift is a sofic subshift if $X=X_{\mathcal{G}}$ for some labeled graph $\mathcal{G}$. Let $\mathcal{G}=(G, \mathcal{L})$ be a labeled graph. $\mathcal{G}$ is called right-resolving if for each vertex $v$ in $G$, all edges leaving $v$ have different labels.

It is known that every sofic shift has a right-resolving graph presentation [6]. Hence, if $X_{\mathcal{G}}$ is a sofic subshift, we will assume that $\mathcal{G}$ is a right-resolving presentation. Notice that $\mathcal{G}$ has $k$ states, which correspond to $k$ total vertices from the graph $G$. Now, define a $k \times k$ adjacency matrix $M_{\mathcal{G}}$ by defining the entries as $m_{i, j}=\sum_{e_{i, j}} \mathcal{L}\left(e_{i, j}\right)$, where $e_{i, j}$ is an edge from vertex $v_{i}$ to $v_{j}$ in the graph $G$. For more information on sofic subshifts and associated graphs, see [6]. We define another $k \times k$ matrix $M_{\mathcal{G}, t}$ by defining the entries as $m_{i, j}^{(t)}=\sum_{e_{i, j}} \mathcal{L}\left(e_{i, j}\right)^{t}$.

Let $\left\{\mathcal{K} ; f_{1}, \ldots, f_{m}\right\}$ be a hyperbolic IFS with $c_{i} d(x, y) \leq d\left(f_{i}(x), f_{i}(y)\right) \leq \bar{c}_{i} d(x, y)$ for $1 \leq i \leq m$ and all $x, y \in \mathcal{K}$. We will define two $k \times k$ matrices, $A_{\mathcal{G}, t}$ and $\bar{A}_{\mathcal{G}, t}$ similar to the matrix $M_{\mathcal{G}, t}$ above. Let $A_{\mathcal{G}, t}$ be defined by the entries $a_{i, j}^{(t)}=\sum_{e_{i, j}} c_{\left(\mathcal{L}\left(e_{i, j}\right)\right)}^{t}$, where $a_{i, j}^{(t)}$ denotes the $(i, j)$-th entry of $A_{\mathcal{G}, t}$. Let $\bar{A}_{\mathcal{G}, t}$ be defined by the entries $\bar{a}_{i, j}^{(t)}=\sum_{e_{i, j}} \bar{c}_{\left(\mathcal{L}\left(e_{i, j}\right)\right)}$.

Lemma 5.1. Let $X_{\mathcal{G}}$ be a sofic subshift, where $\mathcal{G}=(G, \mathcal{L})$. If $G$ has $k$ vertices, then

$$
\frac{1}{k} \sum_{i, j=1}^{k}\left[A_{\mathcal{G}, t}^{n}\right]_{i, j} \leq \sum_{\omega \in W_{n}} c_{\omega}^{t} \leq \sum_{i, j=1}^{k}\left[A_{\mathcal{G}, t}^{n}\right]_{i, j},
$$

where $W_{n}$ denotes all allowable words of length $n$ from $X_{\mathcal{G}}$.

Proof. Let $\omega \in W_{n}$ for some $n \geq 1$. Notice that there may be more than one representation for $\omega$ in $\mathcal{G}$. Since $\sum_{i, j=1}^{k}\left[A_{\mathcal{G}}^{n}\right]_{i j}$ sums contractive factors related to all 
labeled paths of length $n$ in $\mathcal{G}$, then $\sum_{\omega \in W_{n}} c_{\omega}^{t} \leq \sum_{i, j=1}^{k}\left[A_{\mathcal{G}, t}^{n}\right]_{i j}$. Now, if $G$ has $k$ vertices, then $\mathcal{G}$ also has $k$ vertices. By assumption, $\mathcal{G}$ is right-resolving, meaning no two edges leaving the same vertex have the same label. Hence, any $\omega \in W_{n}$ can have at most $k$ representations in $\mathcal{G}$. Therefore, for fixed value $k, \frac{1}{k} \sum_{i, j=1}^{k}\left[A_{\mathcal{G}, t}^{n}\right]_{i, j} \leq$ $\sum_{\omega \in W_{n}} c_{\omega}^{t}$

Theorem 5.2. Let $\left\{\mathcal{K} ; f_{1}, \ldots, f_{m}\right\}$ be a hyperbolic IFS with $c_{i} d(x, y) \leq d\left(f_{i}(x), f_{i}(y)\right) \leq$ $\bar{c}_{i} d(x, y)$ for $1 \leq i \leq m$ and all $x, y \in \mathcal{K}$. Let $X_{\mathcal{G}}$ be a sofic subshift on the alphabet $\mathcal{A}=\{1, \ldots, m\}$ and $\mathcal{F}_{\mathcal{G}}$ be the subfractal defined by the IFS and $X_{\mathcal{G}}$. Suppose $A_{\mathcal{G}}$ is irreducible. If $\rho\left(A_{\mathcal{G}, h}\right)=1=\rho\left(\bar{A}_{\mathcal{G}, H}\right)$, then

$$
h \leq \operatorname{dim}_{H}\left(\mathcal{F}_{\mathcal{G}}\right) \leq H \text { and } h \leq \overline{\operatorname{dim}}_{B}\left(\mathcal{F}_{\mathcal{G}}\right) \leq H .
$$

Proof. By Lemma 5.1, we can rewrite the topological lower and upper pressure function as

$$
\begin{gathered}
P(t)=\lim _{n \rightarrow \infty} \frac{1}{n} \log \left(\sum_{\omega \in W_{n}} c_{\omega}^{t}\right)=\lim _{n \rightarrow \infty} \frac{1}{n} \log \left(\sum_{i, j=1}^{k}\left[A_{\mathcal{G}, t}^{n}\right]_{i, j}\right) \text { and } \\
\bar{P}(t)=\lim _{n \rightarrow \infty} \frac{1}{n} \log \left(\sum_{i, j=1}^{k}\left[\bar{A}_{\mathcal{G}, t}^{n}\right]_{i, j}\right) .
\end{gathered}
$$

The remainder of the proof follows as in Theorem 4.6 and Theorem 4.7.

Remark 4. Similar to Remark 2, the values of $h$ and $H$ such that $P(h)=0=$ $\bar{P}(H)$ also satisfy $\rho\left(A_{\mathcal{G}, h}\right)=1=\rho\left(\bar{A}_{\mathcal{G}, H}\right)$.

Remark 5. Similar to Remark 3, due to known relationships between Hausdorff, packing, upper and lower box dimensions, we also have

$$
h \leq \operatorname{dim}_{P}\left(\mathcal{F}_{\mathcal{G}}\right) \leq H \text { and } h \leq \underline{\operatorname{dim}}_{B}\left(\mathcal{F}_{\mathcal{G}}\right) \leq H .
$$

\section{Generalization to Reducible matrices}

In this section, we will eliminate the irreducibility condition on the matrices in the case of Hausdorff dimension. Consider the case where $A_{\mathcal{G}}$ (or $A_{G}$ if we have an $\mathrm{SFT})$ is a reducible matrix. Let $A$ be a reducible $m \times m(0,1)$ - matrix, and $\mathcal{G}$ be the associated graph. Since $A$ is a reducible matrix, the graph $\mathcal{G}$ is not strongly connected, but it contains a finite number of strongly connected components, say $C_{1}, \ldots, C_{k}$. To each component, we can associate a submatrix $A_{1}, \ldots A_{k}$ where $A_{i}$ is irreducible for $1 \leq i \leq k$. Now, we can simultaneously permute the rows and columns of $A$ to obtain:

$$
\tilde{A}=\left[\begin{array}{ccccc}
A_{k} & 0 & 0 & \cdots & 0 \\
* & A_{k-1} & 0 & \cdots & 0 \\
* & * & A_{k-2} & \cdots & 0 \\
\vdots & \vdots & \vdots & \ddots & \vdots \\
* & * & * & \cdots & A_{1}
\end{array}\right] .
$$


For further details on this process, see [6].

The process used to obtain $\tilde{A}$ from $A$ will not affect the characteristic polynomial, and hence $A$ and $\tilde{A}$ have the same eigenvalues. We can also examine $A^{n}$ and $\tilde{A}^{n}$ similarly; that is, by simultaneously interchanging rows and columns of $A$, each entry of $A^{n}$ will appear in $\tilde{A}^{n}$, although possibly in a different entry position. Hence, we can assume $\sum_{i, j=1}^{m}\left(A^{n}\right)_{i j}=\sum_{i, j=1}^{m}\left(\tilde{A}^{n}\right)_{i j}[6]$. Without loss of generality, we will assume that $A$ is in the form of $\tilde{A}$.

If $A$ is a reducible $m \times m$ matrix with irreducible components $A_{1}, \ldots, A_{k}$, let $A_{i}$ be an $m_{i} \times m_{i}$ matrix for $1 \leq i \leq k$. For $l<k$, we define the set

$$
\operatorname{trn}\left(A_{l}, A_{p}\right)=\left\{a_{i j} \neq 0: \sum_{s=l+1}^{k} m_{s} \leq i \leq \sum_{s=l}^{k} m_{s}, \sum_{s=p+1}^{k} m_{s} \leq j \leq \sum_{s=p}^{k} m_{s}\right\}
$$

of all non-zero entries from $A_{\mathcal{G}}$ corresponding to a transitional edge in $\mathcal{G}$ from component $C_{l}$ associated with $A_{l}$ to the component $C_{p}$ associated with $A_{p}$. Let $W_{t r n}$ denote all finite words in $\Omega_{k}$ corresponding to a transitional edge from the graph $\mathcal{G}$.

Each strongly connected component of the graph, $C_{i}$, corresponds to an irreducible submatrix, $A_{I}$, and a subshift $X_{A_{i}}, 1 \leq i \leq k$. For simplicity, we will talk about the construction of words in $X_{A}$ by using the strongly connected components $C_{i}, 1 \leq i \leq k$ from $\mathcal{G}$. Given the structure of the entire graph $\mathcal{G}$ and direction of the transitional edges, words in $X_{A}$ must begin in a component $C_{i}$, move through components $C_{j}$, and end in component $C_{l}$ where $1 \leq i \leq j \leq l \leq k$. To formalize this in the subshift setting, we introduce the following notation.

For $1 \leq i<j \leq k$, let

$$
X_{A_{i}} \circledast X_{A_{j}}=\left\{\omega \in X_{A}: \omega=\tau a \xi, \text { where } \tau \in W_{*}\left(A_{i}\right), a \in W_{t r n}, \xi \in X_{A_{j}}\right\} .
$$

Similarly, for $1 \leq i_{1}<i_{2}<\cdots<i_{l} \leq k$, we define $X_{A_{i_{1}}} \circledast \cdots \circledast X_{A_{i_{l}}}=\left\{\omega \in X_{A}\right.$ : $\omega=\tau_{1} a_{1} \tau_{2} \cdots a_{l-1} \xi$, where $\tau_{j} \in W_{*}\left(A_{i_{j}}\right), a_{j} \in W_{t r n}$ for $\left.1 \leq j<l, \xi \in X_{A_{i_{l}}}\right\}$.

Lemma 6.1. If $\mathcal{G}$ has $k$ irreducible components for $k \geq 2$, then

$$
X_{\mathcal{G}}=\left(\bigcup_{i=1}^{k} X_{A_{i}}\right) \cup\left(\bigcup_{j=2}^{k} \bigcup_{i_{1}, \ldots, i_{j}=1}^{k} X_{A_{i_{1}}} \circledast \cdots \circledast X_{A_{i_{j}}}\right),
$$

where $i_{l}<i_{l+1}$ for $1 \leq l<j$.

Proof. We will use induction for this argument. If $\mathcal{G}$ has two strongly connected components, $C_{1}$ and $C_{2}$, with at least one transitional edge from $C_{1}$ to $C_{2}$ then it follows that $X_{A_{1}} \cup X_{A_{2}} \cup\left(X_{A_{1}} \circledast X_{A_{2}}\right) \subseteq X_{A_{\mathcal{G}}}$. Now, let $\omega \in X_{A_{\mathcal{G}}}$. Then, $\omega$ must begin in either $C_{1}, C_{2}$, or on a transitional edge. If $\omega$ starts in $C_{2}$, then $\omega \in X_{A_{2}}$ because there are no transitional edges leaving $C_{2}$ in $\mathcal{G}$. If $\omega$ starts on a transitional edge, then $\omega \in\left(X_{A_{1}} \circledast X_{A_{2}}\right)$ because it is of the form $\omega=\tau * a * \xi$ where $\tau$ is the empty word from $W_{*}\left(A_{1}\right)$. If $\omega$ starts in $C_{1}$, then either $\omega \in X_{A_{1}}$ or $\omega \in\left(X_{A_{1}} \circledast X_{A_{2}}\right)$. Hence, we must have

$$
X_{A_{1}} \cup X_{A_{2}} \cup\left(X_{A_{1}} \circledast X_{A_{2}}\right)=X_{A_{\mathcal{G}}} .
$$


Now, assume $\mathcal{G}$ is a connected graph with $k$ strongly connected components, and consider the subgraph, say $\left.\mathcal{G}\right|_{(k-1)}$, consisting of the first $k-1$ components. Assume that

$$
X_{\left.\mathcal{G}\right|_{(k-1)}}=\left(\bigcup_{i=1}^{k-1} X_{A_{i}}\right) \cup\left(\bigcup_{j=2}^{k-1} \bigcup_{i_{1}, \ldots i_{j}=1}^{k-1} X_{A_{i_{1}}} \circledast \cdots \circledast X_{A_{i_{j}}}\right) .
$$

By comparing the graphs $\mathcal{G}$ and $\left.\mathcal{G}\right|_{(k-1)}$ and their corresponding subshifts $X_{\mathcal{G}}$ and $X_{\left.\mathcal{G}\right|_{(k-1)}}$, we can conclude that any word in $X_{\mathcal{G}}-X_{\left.\mathcal{G}\right|_{(k-1)}}$ will end in $C_{k}$. Hence,

$$
X_{A_{\mathcal{G}}}=X_{A_{\mathcal{G}_{k-1}}} \cup X_{A_{k}} \cup\left(\bigcup_{i_{1}, \ldots i_{j}=1}^{k-1} X_{A_{i_{1}}} \circledast \cdots \circledast X_{A_{i_{j}}} \circledast X_{A_{k}}\right),
$$

which satisfies the assertion.

Proposition 6.2. Let $A_{\mathcal{G}}$ be a reducible matrix with irreducible components $A_{1}, \ldots, A_{k}$. Then,

$$
h_{i_{j}} \leq \operatorname{dim}_{H}\left(\mathcal{F}_{X_{A_{i_{1}}} \circledast \cdots \circledast X_{A_{i_{j}}}}\right) \leq H_{i_{j}}
$$

where

$$
h_{i_{j}} \leq \operatorname{dim}_{H}\left(\mathcal{F}_{X_{A_{i_{j}}}}\right) \leq H_{i_{j}}
$$

and $h_{i_{j}}, H_{i_{j}}$ are the bounds from Theorem 5.2.

Proof. Consider a finite word $\tau_{1} a_{1} \tau_{2} a_{2} \ldots \tau_{j-1} a_{j-1}$ where $\tau_{l} \in W_{*}\left(A_{i_{l}}\right)$ for $1 \leq$ $l \leq j-1$ and $a_{l} \in \operatorname{trn}\left(A_{i_{l}}, A_{i_{l}+1}\right)$. For any $n \geq 1$, there are finitely many words $\tau_{l} \in W_{*}\left(A_{i_{l}}\right)$ with $\ell\left(\tau_{l}\right) \leq n$, for all $1 \leq l \leq j$. Hence, there are finitely many words of the form $\tau_{1} a_{1} \cdots \tau_{j-1} a_{j-1}$ of length $n$. So, the collection $S=\left\{\tau_{1} a_{1} \cdots \tau_{j-1} a_{j-1}\right.$ : $\tau_{l} \in W_{*}\left(A_{i_{l}}\right)$ for $\left.1 \leq l \leq j-1, a_{l} \in \operatorname{trn}\left(A_{i_{l}}, A_{i_{l}+1}\right), \ell\left(\tau_{1} a_{1} \cdots \tau_{j-1} a_{j-1}\right)<\infty\right\}$ is at most countable since $W_{*}\left(A_{i_{l}}\right)$ is countable for $i \leq i_{l} \leq k$. For $\omega \in S$, let $\omega X_{A_{i_{j}}}=$

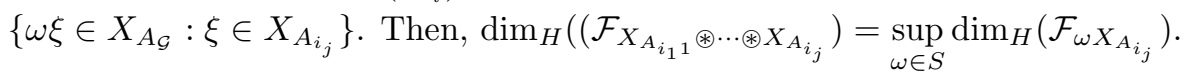

First, notice that $\mathcal{F}_{\omega X_{A_{i}}}=\left\{f_{\omega \xi}(x): \xi \in X_{A_{i}}\right.$ and $\left.x \in \mathcal{K}\right\}$ for any $1 \leq i \leq k$. Recall that $f_{\omega \xi}(x)=f_{\xi} \circ f_{\omega}(x)$ and $f_{\omega}(x) \in \mathcal{K}$ for all $x \in \mathcal{K}$. Hence, $\mathcal{F}_{\omega X_{A_{i}}} \subseteq \mathcal{F}_{\omega_{A_{i}}}$. Hence,

$$
\operatorname{dim}_{H}\left(\mathcal{F}_{\omega X_{A_{i}}}\right) \leq \operatorname{dim}_{H}\left(\mathcal{F}_{X_{A_{i}}}\right) \leq H_{i}
$$

where $H_{i}$ is the bound from Theorem 5.2.

Let $\omega \in S$ with $\ell(\omega)=m$ and $A_{i}$ be an irreducible block in $A$. Consider $\operatorname{dim}_{H}\left(\mathcal{F}_{\omega X_{A_{i}}}\right)$. Although $\omega X_{A_{i}}$ is not necessarily a subshift itself, we can apply similar techniques used to prove Theorem 5.2 to show that the zero of the lower topological pressure function $P_{\omega, i}(t)=\lim _{n \rightarrow \infty} \frac{1}{n} \log \left(\sum_{\tau \in W_{n}\left(\omega X_{A_{i}}\right)} c_{\tau}^{t}\right)$ is a lower bound for 
$\operatorname{dim}_{H}\left(\mathcal{F}_{\omega X_{A_{i}}}\right)$. Notice that

$$
\begin{aligned}
P_{\omega, i}(t) & =\lim _{n \rightarrow \infty} \frac{1}{n} \log \left(\sum_{\tau \in W_{n}\left(\omega X_{A_{i}}\right)} c_{\tau}^{t}\right)=\lim _{n \rightarrow \infty} \frac{1}{n} \log \left(\sum_{\tau \in W_{n-m}\left(X_{A_{i}}\right)} c_{\omega}^{t} c_{\tau}^{t}\right) \\
& =\lim _{n \rightarrow \infty} \frac{1}{n}\left[\log \left(c_{\omega}^{t}\right)+\log \left(\sum_{\tau \in W_{n-m}\left(X_{A_{i}}\right)} c_{\tau}^{t}\right)\right] \\
& =\lim _{n \rightarrow \infty} \frac{1}{n-m} \log \left(\sum_{\tau \in W_{n-m}\left(X_{A_{i}}\right)} c_{\tau}^{t}\right) \\
& =P_{i}(t),
\end{aligned}
$$

where $P_{i}(t)$ is the lower topological pressure function associated with the subfractal $\mathcal{F}_{X_{A_{i}}}$. Hence,

$$
h_{i} \leq \operatorname{dim}_{H}\left(\mathcal{F}_{\omega X_{A_{i}}}\right)
$$

Thus,

$$
\begin{gathered}
\operatorname{dim}_{H}\left(\mathcal{F}_{X_{A_{i_{1} 1}} \circledast \cdots \circledast X_{A_{i_{j}}}}\right)=\sup _{\omega \in S} \operatorname{dim}_{H}\left(\mathcal{F}_{\omega X_{A_{i_{j}}}}\right) \leq H_{i_{j}} \text { and } \\
h_{i_{j}} \leq \operatorname{dim}_{H}\left(\mathcal{F}_{X_{A_{i_{1} 1}} \circledast \cdots \circledast X_{A_{i_{j}}}}\right),
\end{gathered}
$$

where $h_{i_{j}}$ and $H_{i_{j}}$ are the zeros of the upper and lower topological pressure functions $P_{i_{j}}(t)$ and $\bar{P}_{i_{j}}(t)$ with respective the subfractal $\mathcal{F}_{X_{A_{i_{j}}}}$ for some $1 \leq i_{j} \leq k$.

For a similar statement about subshifts with a reducible matrix $A$, we have, by Lemma 6.1,

$$
\mathcal{F}_{X_{A_{\mathcal{G}}}}=\left(\bigcup_{i=1}^{k} \mathcal{F}_{X_{A_{i}}}\right) \cup\left(\bigcup_{j=2}^{k} \bigcup_{1 \leq i_{1}<\cdots<i_{j} \leq k} \mathcal{F}_{X_{A_{i_{1}}} \circledast \cdots \circledast X_{A_{i_{j}}}}\right) .
$$

Thus, by Proposition 6.2, we have the following theorem.

Theorem 6.3. Let $X_{A_{\mathcal{G}}}$ be a sofic subshift associated with matrix $A_{\mathcal{G}}$. Assume $A_{\mathcal{G}}$ has irreducible components $A_{1}, \ldots, A_{k}$. Let $\mathcal{F}_{X_{A_{\mathcal{G}}}}$ and $\mathcal{F}_{X_{A_{i}}}$ denote the sub-fractals associated with the subshifts $X_{A_{\mathcal{G}}}$ and $X_{A_{i}}$, respectively. Then,

$$
\max _{1 \leq i \leq k}\left\{h_{i}\right\} \leq \operatorname{dim}_{H}\left(\mathcal{F}_{X_{A_{\mathcal{G}}}}\right) \leq \max _{1 \leq i \leq k}\left\{H_{i}\right\},
$$

where $P_{i}\left(h_{i}\right)=0=\bar{P}_{i}\left(H_{i}\right)$ given in Theorem 5.2 for all $1 \leq i \leq k$.

Acknowledgements The author acknowledges ND-EPSCoR and NSF grant 1355466 for funding her research. She thanks her advisor, Doğan Çömez for his guidance and many helpful conversations during the construction of this paper. She is grateful to Michael P. Cohen for his edits and especially useful comments. She also thanks Mrinal Kanti Roychowdhury and Azer Akhmedov for their comments on an earlier preprint. 


\section{REFERENCES}

[1] Gerald Edgar, Measure, topology, and fractal geometry, second ed., Undergraduate Texts in Mathematics, Springer, New York, 2008.

[2] David B. Ellis and Michael G. Branton, Non-self-similar attractors of hyperbolic iterated function systems, Dynamical systems (College Park, MD, 1986-87), Lecture Notes in Math., vol. 1342, Springer, Berlin, 1988, pp. 158-171.

[3] Kenneth Falconer, Techniques in fractal geometry, John Wiley \& Sons Ltd., Chichester, 1997.

[4] Kenneth Falconer, Fractal geometry, second ed., John Wiley \& Sons, Inc., Hoboken, NJ, 2003.

[5] John E. Hutchinson, Fractals and self-similarity, Indiana Univ. Math. J. 30 (1981), no.5, 713-747.

[6] Douglas Lind and Brian Marcus, An introduction to symbolic dynamics and coding, Cambridge University Press, Cambridge, 1995.

[7] Yakov B. Pesin, Dimension theory in dynamical systems, Chicago Lectures in Mathematics, University of Chicago Press, Chicago, IL, 1997.

[8] Mrinal Kanti Roychowdhury, Hausdorff and upper box dimension estimate of hyperbolic recurrent sets, Israel J. Math. 201 (2014), no. 2, 507-523.

North Dakota State University, PO Box 6050, Fargo, ND 58108

E-mail address: elizabeth.sattler.1@ndsu.edu 